\title{
Le tau et le tau-b de Kendall pour la corrélation de variables ordinales simples ou catégorielles
}

\author{
L. Laurencelle, \\ Université du Québec à Trois-Rivières
}

Dans le langage de tous les jours, l'expression «corrélation entre deux variables » est entendue et bien comprise de manière générale : c'est un lien, un rapport de correspondance grâce auquel la variation d'un attribut peut être associée à la variation d'un autre attribut.

En fait, ce concept général de corrélation - on devrait plutôt employer le terme « association »- en recouvre trois qui, dans le langage plus rigoureux des interprétations scientifiques, ont chacun leur formulation mathématique et leur acception spécifiques. Ces trois types d'association sont :

une relation non spécifique : $\mathrm{Y}$ a-t-il un lien, une influence quelconque - causale, indirecte, mutuelle - entre deux caractéristiques observées ? Des exemples de ce type de relation abondent : le niveau moyen de réponse de deux ou plusieurs groupes, selon le traitement particulier auquel ont été soumis leurs participants, l'incidence de telle maladie selon la région géographique ou la race, la force musculaire selon le sexe, etc. Ces relations impliquent ordinairement une variable catégorielle (ou qualitative), tels le sexe, la condition expérimentale, la race, et une variable dépendante qui peut être ou mesurée (la force, le degré de réussite), ou observée (l'option politique, la présence de maladie). L'arsenal disponible pour l'évaluation statistique de ces relations est nombreux et diversifié, les outils les plus courants étant les tests $t$ de Student, l'analyse de variance et le test du Khi-deux pour tableaux de fréquences. Laurencelle (2005) a considéré le cas d'une association non monotone entre deux variables continues.

une relation linéaire: La variation d'une grandeur $Y$ donnée est-elle proportionnelle à la variation d'une autre grandeur $X$, peut-on établir une règle de correspondance linéaire entre les deux? Cette relation paradigmatique, représentée par l'équation « $\mathrm{Y}=\mathrm{bX}+\mathrm{a}$ », fournit souvent le premier pas vers une modélisation d'un phénomène et elle en constitue aussi l'aboutissement rêvé, parfois sous une forme augmentée telle que « $Y=f(X) »$, où $f$ est une fonction, linéaire ou linéarisable, de $X$. Température et longueur d'une tige de fer, réussite scolaire et quotient intellectuel, fréquence cardiaque maximale et âge sont trois cas de relation linéaire simple à peu près démontrés. Le coefficient de corrélation linéaire, diversement attribué à Bravais, Galton et K. Pearson, en est la mesure directe, sous la forme simplifiée

$$
r \propto \Sigma\left(X_{i}-\mu x\right)\left(Y_{i}-\mu \gamma\right) .
$$

La réécriture de l'équation « $Y=b X+a$ » sous la forme « $(Y-$ $\mu \gamma)=b(X-\mu x) »$ montre à l'évidence que cette corrélation quantifie le degré de correspondance proportionnelle entre $X$ et $Y$ et qu'elle est donc intimement solidaire de la métrique de ces deux variables. L'analyse de régression, la régression polynomiale et le modèle linéaire général comptent parmi les techniques d'évaluation privilégiées pour ce type de relation.

une relation monotone: Intermédiaire entre la relation linéaire décrite ci-dessus et une relation non spécifique, qui n'implique aucune concordance régulière entre $X$ et $Y$, $\mathrm{s}$ 'intercale la relation dite monotone. $Y$ a-t-il une fatigue, une diminution d'énergie chez les travailleurs $d u$ premier jusqu'au cinquième jour d'une semaine de travail? Les enfants dont la diète comporte une dose plus importante de fruits et légumes sont-ils moins sujets à certaines maladies? L'incidence d'accidents vasculocérébraux augmente-t-elle avec le cholestérol sanguin total ? Les relations de ce type se subdivisent en deux sous-catégories, selon la nature des variables concernées :

- $\quad$ soit les variables $X$ et $Y$ concernées sont toutes deux mesurées et reflètent des grandeurs (continues), alors que la relation supposée entre elles est monotone plutôt que métrique (p. ex. l'espérance de vie vs le revenu moyen);

- $\quad$ soit au moins l'une des variables est ordinale (p. ex. l'échelle de Mohs pour la dureté d'un matériau) ou catégorielle ordonnée, la variable catégorielle regroupant 
ordinairement plus d'un élément (p. ex. le niveau de scolarité).

Par relation monotone, on entend une correspondance entre les variables $X$ et $Y$ telle qu'une inégalité dans une variable entraîne une inégalité dans l'autre, la relation étant positive si $X_{i}>X_{j}$ entraîne habituellement $Y_{i}>Y_{j}$ ou négative si $X_{i}>X_{j}$ entraîne $Y_{i}<Y_{j}$. Ce type de relation, sans modèle spécifié et qui concerne des variables sans métrique ou à métrique floue (pour ainsi dire), n'a guère été achalandé par les chercheurs, et les techniques disponibles sont peu nombreuses et comparativement peu connues. D'un côté, suite à Jonckeere (1954), Barlow, Bartholomew, Bremmer et Brunk (1972) mirent au point un test de «variation monotone », applicable dans le contexte de l'analyse de variance, l'une des variables étant catégorisée (voir Laurencelle 1993, Laurencelle et Dupuis 2000, Mukerjee 1988). De l'autre, Kendall (Kendall et Gibbons 1990) a consacré son fameux indice «tau », lequel quantifie le degré de concordance monotone entre deux variables ordinales, qu'elles soient continues (indice $\tau$, ou tau) ou catégorielles (indice $\tau \mathrm{b}$, ou tau-b).

Comme le fera voir l'exposé plus loin, le tau de Kendall a, sur la corrélation $r$ de Pearson, deux avantages décisifs, celui de transcender la métrique des variables mesurées et celui d'écarter la nécessité d'un modèle paramétrique de relation (comme le modèle linéaire), ce qui en fait un indice authentiquement non paramétrique, approprié aux variables de type ordinal. Tel n'est pas le cas du coefficient « rho » de Spearman (noté ici $r s$ ), lequel invoque un modèle linéaire strict, c'est-à-dire un calcul ordinaire du $r$ de Pearson appliqué sur les rangs des variables considérées.

Quant à la corrélation entre variables ordinales catégorielles, elle mérite d'être ré-examinée avec attention. Dans un exemple type (tel que celui présenté plus loin, voir Calcul du $\tau$ b), des observations $X, Y$ en nombre important $(n)$ sont réparties en quelques catégories d'une variable $(k x)$ et de l'autre $(k r)$, le nombre moyen de valeurs partagées ou égales dans chaque variable $(n / k x, n / k r)$ étant élevé. Ce contexte statistique conduit naturellement à la constitution d'un tableau croisé, d'ordre $k x \times k y$, qui synthétise l'information: chaque cellule contient alors le nombre d'observations inscrites concomitamment dans une catégorie d'une variable $\left(X_{i}\right)$ et de l'autre $\left(Y_{j}\right)$. Or, l'outil traditionnel appliqué au traitement d'un tableau croisé de fréquences tel que celui-là est le test du khi-deux, le khideux d'interaction, qui permet de rejeter l'hypothèse de l'indépendance stochastique des deux variables mesurées. Toutefois, comme l'ont bien montré Burr (1960) et Somers (1962), grâce à des exemples et contre-exemples, le khi-deux

\footnotetext{
${ }^{1}$ Le verbe «entraîner » est proposé ici dans une acception descriptive, statistique, et ne présuppose pas le concept de causalité ou d'influence.
}

d'interaction ne convient pas à cet usage : la sanction que donne le khi-deux est générale et ne porte pas spécifiquement sur l'hypothèse d'une relation monotone entre les variables $X$ et $Y$. Pour cette raison, un khi-deux significatif $\mathrm{n}^{\prime}$ indique pas forcément la présence d'une relation monotone, et la présence d'une relation monotone statistiquement sérieuse (p. ex. d'après un argument de probabilité direct) pourrait passer inaperçue au khi-deux en raison de sa faible puissance. Le test approprié, le tau-b (Kendall et Gibbons 1990), est une variante simple du tau, qui tient compte du caractère catégoriel des variables observées et pour lequel un calcul à partir du tableau croisé de fréquences est aussi suggéré. Le lecteur trouvera une variante asymétrique du tau-b dans Somers (1962).

Les variables catégorielles, qu'on désigne parfois «qualitatives », apparaissent principalement dans les données d'enquête et de sondage : épidémiologie, enquête de consommation, opinion publique, et leur traitement coutumier passe par le progiciel SPSS ou par d'autres moyens semblables. Les interdépendances possibles entre variables sont ordinairement testées par le khi-deux d'interaction, même dans les cas où les deux variables concernées sont ordonnées : une pléthore de publications peut être citée à l'appui. Or, tel que mentionné précédemment et dans ce contexte :

1- l'obtention d'un khi-deux significatif indique que les deux variables sont statistiquement non indépendantes ${ }^{2}$, non pas qu'elles ont un rapport de relation monotone l'une avec l'autre ;

2- l'obtention d'un khi-deux non significatif n'implique pas qu'il n'y ait pas de relation monotone significative entre les deux variables.

C'est pourquoi il nous est apparu important de présenter à nouveau cet outil particulier qu'est le tau de Kendall, et plus spécialement de réactualiser le tau-b pour la corrélation entre deux variables ordinales catégorielles.

\section{Calcul du $\tau$, avec exemple}

La formule classique du $\tau$ de Kendall, applicable à des variables ordinales simples, sans catégorisations ni valeurs égales, est :

$$
\tau=(C-D) /\left(\begin{array}{l}
n \\
2
\end{array}\right),
$$

où $\mathrm{C}=$ nombre d'inégalités concordantes $\left[\mathrm{p} . \mathrm{ex} . X_{i}>X_{j}\right.$ et $Y_{i}>$ $\left.Y_{j}\right] ; \mathrm{D}=$ nombre d'inégalités discordantes [ p.ex. $X_{i}>X_{j}$ et $\left.Y_{i}<Y_{j}\right] ; n=$ nombre de paires $X, Y$.

2 Dans le test du khi-deux d'interaction, la composante additive du test, " $\left(n_{i, j}-n_{i} \times n_{j}\right)^{2} /\left(n_{i} \times n_{j}\right)$ », reste inchangée sous toute permutation des rangées ou des colonnes du tableau, de sorte que la valeur du khi-deux reste la même, la corrélation (monotone) dépendant quant à elle de l'ordre des catégories tel qu'observé. 
La différence $(C-D)$, au numérateur de (1), peut être obtenue par la somme $\sum_{i, j}$ signe $\left\{\left(X_{i}-X_{j}\right)\left(Y_{i}-Y_{j}\right)\right\}$, effectuée sur les $\left(\begin{array}{l}n \\ 2\end{array}\right)=1 / 2 n(n-1)$ paires possibles d'observations, où $\operatorname{signe}(z)=-1,0$ ou 1 selon que $z<0, z=0$ ou $z>0$ respectivement.

Une autre méthode, indiquée par exemple dans Siegel et Castellan (1988) pour le calcul manuel, consiste en deux étapes. En premier, il s'agit de placer la série $X, Y$ en ordre croissant des valeurs $X$. En second, pour chacune des observations $Y_{i}$, de $i=1$ à $i=n-1$, il faut compter le nombre de valeurs $Y_{j}$ suivantes qui sont plus grandes (c.-à-d. $Y_{j}>Y_{i}$ pour $j>i)$, moins le nombre de valeurs plus petites $\left(Y_{j}<Y_{i}\right)$, le total donnant $\mathrm{C}-\mathrm{D}$. La même formule (1) s'applique. L'exemple 1 illustre les calculs.

\begin{tabular}{c|cccccc}
\multicolumn{8}{c}{ Exemple 1. Données ordinales distinctes } \\
\hline$X$ & 10 & 4 & 16 & 5 & 13 & 14 \\
$Y$ & 17 & 14 & 20 & 8 & 11 & 23 \\
\hline
\end{tabular}

L'exemple nous met en présence de $n=6$ paires $X, Y$; les données de chaque variable sont toutes distinctes. Ces $n=6$ valeurs donnent lieu à $\left(\begin{array}{l}n \\ 2\end{array}\right)=15$ comparaisons. La liste des comparaisons suit :

\begin{tabular}{cccc}
\hline compar. en $\mathrm{X}$ & compar. en $\mathrm{Y}$ & concord. & discord. \\
\hline $10>4$ & $17>14$ & $\mathrm{~T}$ & \\
$10<16$ & $17<20$ & $\mathrm{~T}$ & \\
$10>5$ & $17>8$ & $\mathrm{~T}$ & $\mathrm{~T}$ \\
$10<13$ & $17>11$ & & \\
$10<14$ & $17<23$ & $\mathrm{~T}$ & \\
$4<16$ & $14<20$ & $\mathrm{~T}$ & $\mathrm{~T}$ \\
$4<5$ & $14>8$ & & $\mathrm{~T}$ \\
$4<13$ & $14>11$ & & \\
$4<14$ & $14<23$ & $\mathrm{~T}$ & \\
$16>5$ & $10>8$ & $\mathrm{~T}$ & $\mathrm{~T}$ \\
$16>13$ & $20>11$ & $\mathrm{~T}$ & \\
$16>14$ & $20<23$ & & $\mathrm{~T}$ \\
$5<13$ & $8<11$ & $\mathrm{~T}$ & $\mathrm{D}=4$ \\
$5<14$ & $8<23$ & $\mathrm{~T}$ & $\mathrm{~T}$ \\
$13<14$ & $11<23$ & $\mathrm{C}=11$ & \\
& & &
\end{tabular}

L'analyse donne $\mathrm{C}=11$ comparaisons concordantes, c'est-à-dire des comparaisons pour lesquelles l'inégalité observée en $X$ est de même signe que celle observée en $Y$, et $\mathrm{D}=4$ comparaisons discordantes; notons que $\mathrm{C}+\mathrm{D}=\left(\begin{array}{l}n \\ 2\end{array}\right)=$ 15. Appliquant la formule (1), nous obtenons donc :

$$
\begin{aligned}
\tau= & (11-4) /\left(\begin{array}{l}
6 \\
2
\end{array}\right) \\
= & 7 / 15 \\
= & 0,467 .
\end{aligned}
$$

Pour le procédé «manuel» suggéré par Siegel et
Castellan (1988), nous devons d'abord placer les observations $X$ en ordre de valeurs croissantes, accompagnées des $Y_{i}$ correspondantes. Ensuite, pour chaque $Y_{i}$, il nous faut ensuite compter, en plus ou en moins, le nombre d'observations $Y_{j}$ suivantes (pour lesquelles $j>i$ ) selon qu'elles sont plus fortes ou moins fortes que $Y_{i}$ : le total donne $\mathrm{C}-\mathrm{D}$, tel qu'illustré par le tableau suivant ${ }^{3}$.

\begin{tabular}{lll}
\hline $\mathrm{X}$ & $\mathrm{Y}$ & $\sum \operatorname{signe}\left(\mathrm{Y}_{\mathrm{j}}-\mathrm{Y}_{\mathrm{i}}\right)$ \\
\hline 4 & 14 & $3-2$ \\
5 & 8 & $4-0$ \\
10 & 17 & $2-1$ \\
13 & 11 & $2-0$ \\
14 & 23 & $0-1$ \\
16 & 20 & \\
\hline
\end{tabular}

Prenons, par exemple, la première valeur de $Y, Y_{1}=14$; dans la série des $Y_{j}$ qui la suivent, les valeurs 17, 23 et 20 sont supérieures (>14), tandis que 8 et 11 sont inférieures, d'où le compte net est de 1, La compilation pour $Y_{2}$ et les $Y$ suivantes donne un total $(C-D)$ de 7 . L'application de la formule (1) fournit encore :

$$
\begin{aligned}
\tau & =7 / 15 \\
& =0,467 .
\end{aligned}
$$

Nous considérons plus loin la distribution de probabilité de cet indice et la procédure de test qui lui convient.

\section{Calcul $d u \tau_{b}$, avec exemples}

L'indice tau-b (noté $\tau \mathrm{b}$ ) est une extension du $\tau$ de Kendall pour données ordinales non distinctes, c'est dire qu'il s'applique de façon particulièrement idoine aux variables ordinales catégorielles. Pour le calcul, la règle des signes, $\Sigma_{i, j}$ signe $\left\{\left(X_{i}-X_{j}\right)\left(Y_{i}-Y_{j}\right)\right\}$, s'applique encore, le cas de valeurs égales, soit $X_{i}=X_{j}$ ou $Y_{i}=Y_{j}$, aboutissant à un résultat de 0 .

La formule à retenir est :

$$
\tau_{\mathrm{b}}=(\mathrm{C}-\mathrm{D}) / \mathrm{Den}
$$

où :

$$
\text { Den }=1 / 2 \sqrt{\left[n(n-1)-\mathrm{U}_{X}\left[n(n-1)-\mathrm{U}_{Y}\right]\right.}
$$

et :

$$
\begin{aligned}
& \mathrm{U}_{X}=\Sigma \mathbf{u}_{X}\left(\mathbf{u}_{X}-1\right) ， \\
& \mathrm{U}_{Y}=\Sigma \mathbf{u}_{Y}\left(\mathbf{u}_{Y}-1\right) ;
\end{aligned}
$$

les symboles « $u_{x}$ » et « $u r$ » dénotent le nombre d'apparitions de chaque valeur $X$ et chaque valeur $Y$ respectivement. Noter que la formule (1) est un cas particulier de celle-ci, où $\mathbf{u}_{X}=\mathbf{u}_{Y}=1$ pour tous les $X$ et tous les $Y$ (chaque valeur n'apparaissant qu'une fois). Nous

${ }^{3}$ L'astuce ici vient de ce que les données ont été ordonnées selon $X$, de sorte qu'il n'est pas utile de tenir compte du signe des inégalités en $X$ (qui sont alors toutes positives). 
présentons deux exemples de calcul, un premier, plus simple, à partir d'une courte série $X, Y$, et un second, basé sur un tableau croisé de fréquences.

Calcul sur une série (exemple 2). L'exemple 2, ci-dessous, servira d'illustration.

Exemple 2. Données ordinales avec égalités

\begin{tabular}{l|llllll}
\hline $\mathrm{X}$ & 10 & 18 & 18 & 20 & 26 & 36 \\
$\mathrm{Y}$ & 12 & 19 & 12 & 31 & 29 & 33 \\
\hline
\end{tabular}

Le procédé de Siegel et Castellan (1988), hélas, ne s'applique pas ici, en raison des permutations possibles des paires comportant des valeurs égales. Il faut plutôt recourir laborieusement aux comparaisons par paires. Parmi les $\left(\begin{array}{l}6 \\ 2\end{array}\right)=$ 15 paires générées, la série $X, Y$ de l'exemple 2 contient $C=$ 12 paires concordantes, $\mathrm{D}=1$ paire discordante et 2 paires avec égalités, d'où résulte le numérateur $C-D=11$. Pour le dénominateur (3), les quantités $U_{X}$ et $U_{Y}$ sont nourries par le nombre de valeurs ( $X$ ou $Y$ ) apparaissant plus d'une fois. Ici, en $X$, seule la valeur « 18 » apparaît 2 fois, i.e. u18 $=2$, d'où $\mathrm{U}_{X}=2 \times(2-1)=2$; de même pour la valeur « 12 » en $Y$, d'où $\mathrm{U}_{Y}=2$. Les autres valeurs de $X$ ou $Y, n^{\prime}$ apparaissant $q u^{\prime}$ une fois (i.e. pour $X=10, \mathrm{u}_{10}=1$ ), n'ajoutent rien à leur somme respective $U_{x}$ et $U_{\gamma}$. Le dénominateur devient donc :

$$
\text { Den }=1 / 2 \sqrt{(6 \times 5-2)(6 \times 5-2)}=14 \text {. }
$$

Nous obtenons enfin :

$$
\tau \mathrm{b}=11 / 14
$$

Exemple 3. Données ordinales catégorielles

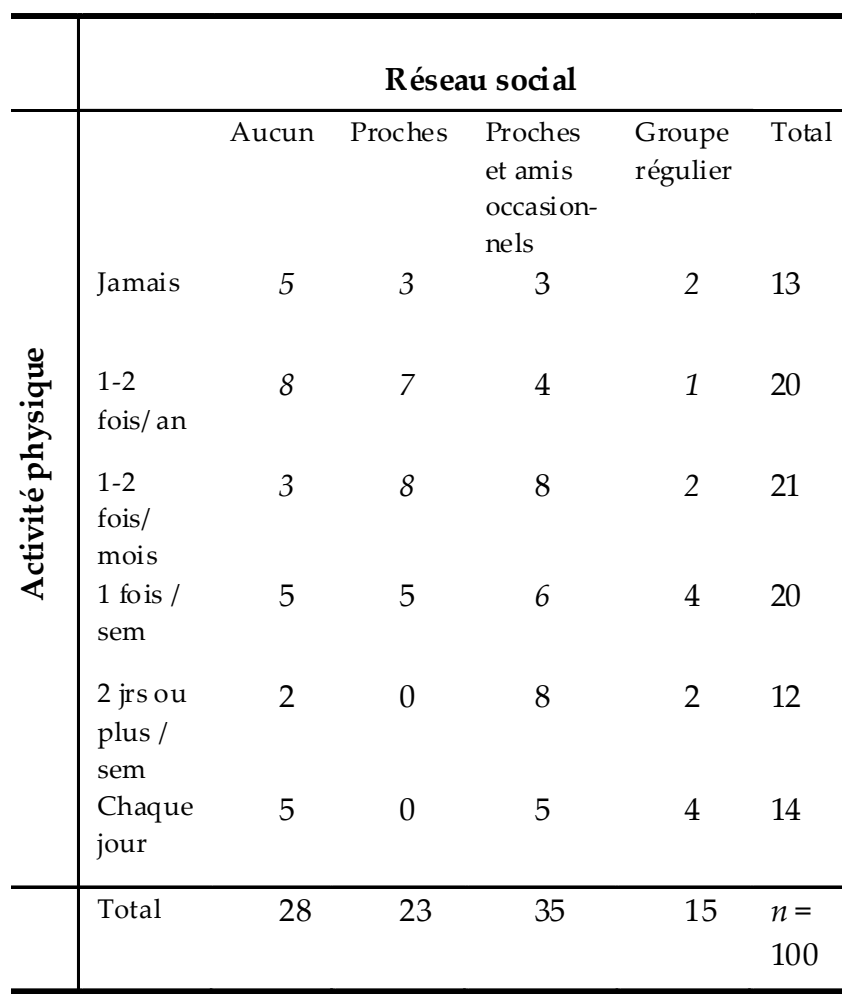

$$
=0,786 \text {. }
$$

Calcul sur un tableau $\mathbf{X} \times \mathbf{Y}$ (exemple 3$)$. Le calcul du $\tau_{b}$ à partir d'un tableau croisé de fréquences met en oeuvre les mêmes principes que ceux pratiqués dans l'exemple précédent, seul le format de la compilation change. Nous baserons notre explication sur l'exemple 3, ci-dessous. Au total, $100(=n)$ personnes ont été interrogées quant à leur réseau social (au travail et hors travail) et leur pratique d'activités physiques. En admettant que les catégories d'activité physique croissent en intensité (depuis «jamais » jusqu'à «chaque jour»), tout comme les catégories du réseau social en importance (depuis «aucun (réseau)» jusqu'à "groupe régulier»), nous nous demandons s'il y a un lien de corrélation entre l'une et l'autre variable.

Le tableau, comme on voit, se présente avec des catégories de "valeurs », croissantes de gauche à droite et de haut en bas: le dénombrement des inégalités concordantes et discordantes en tire profit. Prenons, par exemple, le cas des personnes pratiquant « 1 fois / sem » et ayant comme réseau des "Proches et amis occasionnels »: leur nombre est de 6. Alors, dans le tableau, toutes les observations qui se situent en haut et à gauche sont concordantes (leur nombre est $5+3+8+7+3+8=34$ ), et celles situées en haut et à droite sont discordantes (leur nombre est $2+1+2=5$ ): la portion nette d'inégalités relative à cette cellule est donc $6 \times(34-5)=174$.

Le calcul systématique peut être noté comme suit (depuis la $2^{\mathrm{e}}$ ligne du tableau) :

\begin{tabular}{lllll}
\hline $8 \times(0-8)+$ & $7 \times(5-5)+$ & $4 \times(8-2)+$ & $1 \times(11-0)=$ & -29 \\
$3 \times(0-20)+$ & $8 \times(13-10)+$ & $8 \times(23-3)+$ & $2 \times(30-0)=$ & 184 \\
$5 \times(0-38)+$ & $5 \times(16-20)+$ & $6 \times(34-5)+$ & $4 \times(49-0)=$ & 160 \\
$2 \times(0-53)+$ & $0 \times(21-30)+$ & $8 \times(44-9)+$ & $2 \times(65-0)=$ & 304 \\
$5 \times(0-63)+$ & $0 \times(23-40)+$ & $5 \times(46-11)+$ & $4 \times(75-0)=$ & 160 \\
\hline & & $\mathrm{C}-\mathrm{D}($ total $)=$ & 779 \\
\hline
\end{tabular}

Le numérateur du $\tau_{b}$ est donc $C-D=779$. Quant $a u$ dénominateur, il faut d'abord établir les quantités $U_{X}$ et $U_{Y}$, ce à partir des totaux de rangées $(X)$ et colonnes $(Y)$ du tableau croisé ; ces totaux indiquent le nombre de "valeurs égales » pour chaque valeur $X$ et $Y$. Nous avons donc :

$$
\begin{aligned}
& U_{X}=13 \times(13-1)+20 \times(20-1)+\ldots+14 \times(14-1)=1650, \\
& U_{Y}=28 \times(28-1)+23 \times(23-1)+\ldots+15 \times(15-1)=2594 .
\end{aligned}
$$

d'où le dénominateur (3) s'obtient par :

$$
\text { Den }=1 / 2 \sqrt{(100 \times 99-1650)(100 \times 99-2594)}
$$

$$
\approx 3881,833 \text {. }
$$

La valeur du tau-b est enfin :

$$
\begin{aligned}
\tau_{\mathrm{b}}= & 779 / 3881,833 \\
& \approx 0,2007 .
\end{aligned}
$$

Nous considérons maintenant les propriétés statistiques 
(distribution et moments) des indices $\tau$ et $\tau$, et présentons des tests de significativité appropriés.

\section{Distribution, moments, test et approximation pour $\tau$}

Le numérateur de $\tau, s=\mathrm{C}-\mathrm{D}$, peut prendre $1 / 2 n(n-1)+1$ valeurs différentes, qui courent de $s=-1 / 2 n(n-1)$ à $s=1 / 2 n(n-1)$ par incréments de 2 ; par exemple, pour $n=3$, s peut prendre les valeurs $-3,-1,1,3$. De plus, le nombre total d'arrangements des séries $X, Y$ générant ces valeurs de $s$ est $n$ !, chacun ayant une probabilité de $1 / n$ !. Kendall et Gibbons (1990) détaillent le procédé récursif suivant, qui permet de générer la distribution de probabilité de $\tau$ sous des permutations au hasard des séries. Soit $n=2$, les valeurs $s=-1$ et 1 (correspondant aux séries 2-1 et 1-2), et leurs fréquences relatives $\left\{\begin{array}{ll}1 & 1\end{array}\right\}$. Ainsi, la valeur $s=-1$ (correspondant à $\tau=-1$ ) a une probabilité de $1 / 2 !=0,50$. Pour générer les fréquences pour des séries de $n$ à partir de $n-1$, il faut superposer $n$ fois la série antécédente, en la décalant chaque fois d'une position à droite, puis additionner les fréquences qui sont en vis-à-vis. Ainsi, pour $n=3$ (depuis $n-1=2$ ), nous avons :

\begin{tabular}{llll}
\hline 1 & 1 & & \\
& 1 & 1 & \\
& & 1 & 1 \\
\hline 1 & 2 & 2 & 1 \\
\hline
\end{tabular}

Les fréquences " $\begin{array}{lllll}1 & 2 & 2 & 1\end{array}$, qui représentent 3 ! = 6 arrangements, correspondent aux valeurs $s=-3,-1,1,3$. Pour aller à $n=4$, nous reprenons pareillement la série de $n-$ $1=3$, et :

\begin{tabular}{lllllll}
\hline 1 & 2 & 2 & 1 & & & \\
& 1 & 2 & 2 & 1 & & \\
& & 1 & 2 & 2 & 1 & \\
& & & 1 & 2 & 2 & 1 \\
\hline 1 & 3 & 5 & 6 & 5 & 3 & 1 \\
\hline
\end{tabular}

Ces fréquences, de total $4 !=24$, correspondant à $s=-6,-4,-$ $2,0,2,4,6$. Kendall et Gibbons (op. cit.) proposent un second procédé récursif de calcul, que nous omettons ici.

Les moments de la distribution de $\tau$ sont :

$$
\begin{gathered}
\mu=0 \\
\sigma^{2}=(4 n+10) / 9 n(n-1) \\
\gamma_{1}=0 \\
\gamma_{2}=-\left(\frac{6}{5}\right)^{2} \frac{6 n^{3}+21 n^{2}+31 n+31}{n(n-1)(2 n+5)^{2}}
\end{gathered}
$$

La distribution est évidemment symétrique $\left(\gamma_{1}=0\right)$. Son indice d'aplatissement, $\gamma_{2}$, avoisine de $-2 /(n-1)$, se rapprochant rapidement de 0 avec $n$ croissant. Ces deux caractéristiques promettent une approximation normale d'un certain succès.
L'approximation de la distribution de $\tau$ par une normale standard passe par la formule :

$$
z=[\tau \pm K / n(n-1)] / \sigma, K=2,
$$

laquelle comporte une correction de continuité (voir aussi Burr 1960). Best (1973), qui a étudié cette approximation, propose qu'elle est déficiente pour $n \leq 40$ et il publie des valeurs critiques exactes allant jusqu'à $n=100$. Nous avons étudié une approximation plus serrée, grâce à la loi Bêta symétrique, $\beta(p, p)$ qui reproduit à peu près l'aplatissement de $\tau$ avec $p=1,39892 n-1,56354$; à notre surprise, l'approximation par la Bêta s'est révélée décevante, voire parfois inférieure à la normale. Nous nous accordons partiellement avec Best, à la nuance suivante. Les valeurs critiques exactes semblent requises (aux seuils de signification habituels) pour $n \leq 29$. Pour $n \geq 30$, l'approximation (6) s'appliquerait mais avec une correction utilisant $K=3$ plutôt que $K=2 .{ }^{4}$ Dès $n \geq 100$, l'usager peut utiliser sans crainte l'approximation (6) telle que présentée. À toutes fins utiles, nous incluons en annexe notre propre table de valeurs critiques exactes du $\tau$, pour $n=4$ à 100 et les seuils de signification $0,10,0,05,0,025,0,01$ et 0,005 .

L'exemple 1, ci-dessus, comportait une série de $n=6$ données $X, Y$, avec un indice $\tau=0,467$ ( $=7 / 15$ ). Pour $n=6$, les moments du $\tau$ sont $\mu=0, \sigma^{2}=34 / 270 \approx 0,1259$ ( $\left.\sigma \approx 0,355\right)$, $\gamma_{1}=0$ et $\gamma_{2} \approx-0,3769$. Comme les fréquences ponctuelle et cumulative pour $s=7$ sont 49 et 671 , le $\tau=7 / 15 \approx 0,467$ obtient une probabilité exacte de $(720-671+49) / 6 ! \approx 0,136$. La table 1 , en annexe, propose $\pm 0,867$ comme valeurs critiques au seuil de $5 \%$ bilatéral. Quant à l'approximation normale (équation 6 , avec $K=3$ ), guère utile ici en raison du petit $n=6$, elle donne :

$$
\begin{aligned}
z & =(0,467-3 /(6 \times 5)) / 0,355 \\
& \approx 1,034,
\end{aligned}
$$

valeur dont la probabilité extrême (sous la loi normale standard) est 0,151 .

\section{Moments, test et approximation pour $\tau_{b}$}

Considérablement plus complexe, la distribution du tau$\mathrm{b}$ est aussi moins documentée. Notant encore $s=\mathrm{C}-\mathrm{D}$ pour le numérateur de l'indice to (2), Burr (1960) détaille la distribution de probabilité pour tous les arrangements (avec égalités) possibles pour $n \leq 6$, tandis que Sillito (1947) le fait pour $n \leq 10$ mais en ne considérant que des égalités de 2 ou 3 composantes des séries. Valz, McLeod et Thompson (1995) tentent, quant à eux, d'établir une fonction génératrice approximative de la distribution $d u \tau b$, sans grand succès, l'approximation normale restant la première en lice pour des tailles $n$ suffisantes.

Rappelant la formule (2) sous la forme $\tau_{b}=s /$ Den, Kendall et Gibbons (1990) démontrent les deux premiers

4 La platykurtose $\left(\gamma_{2}<0\right)$ encore présente dans la distribution explique sans doute en partie ce changement. 
moments :

$$
\mu(\mathrm{s})=0
$$

$\operatorname{var}(s)=\left[n(n-1)(2 n+5)-\mathrm{V}_{X}-\mathrm{V}_{Y}\right] / 18+\mathrm{WXW}_{\mathrm{X}} /[9 n(n-1)(n-2)]$ $+\mathrm{UX}_{X} /[2 n(n-1)]$

où :

$$
\begin{aligned}
& \mathrm{Ux}_{\mathrm{X}}=\Sigma \mathrm{ux}(\mathrm{ux}-1) ; \mathrm{U}_{y}=\Sigma \mathrm{u} \mathrm{u}(\mathrm{u} \gamma-1) \\
& \mathrm{V}_{X}=\Sigma \mathrm{ux}_{\mathrm{X}}\left(\mathrm{u}_{X}-1\right)\left(2 \mathrm{uX}_{\mathrm{X}}+5\right) ; \mathrm{V}_{Y}=\Sigma \mathrm{u}_{Y}(\mathrm{u} Y-1)(2 \mathrm{u} Y+5)
\end{aligned}
$$

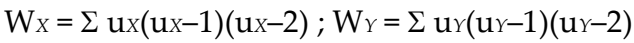

d'où, évidemment :

$$
\begin{gathered}
\mu\left(\tau_{\mathrm{b}}\right)=0 \\
\operatorname{var}(\tau \mathrm{b})=\operatorname{var}(s) /(\text { Den })^{2}
\end{gathered}
$$

Comme on peut imaginer et hormis les exceptions déjà mentionnées, il n'existe pas et il n'est pas raisonnable de demander de tables de valeurs critiques exactes pour des séries de tailles $n$ utiles ${ }^{5}$. Le critère distributionnel (par permutations aléatoires de la série $Y$, voir p. ex. Edgington 1980 ou Laurencelle 2001) peut suppléer aux tables dans le cas de tailles $n$ modestes; au delà, l'approximation normale (6), avec $K=2$, est recommandée.

Pour l'exemple 2, avec une série $X, Y$ de $n=6$ éléments, les ingrédients (8a)-(8c) de la variance sont: $U_{X}=2, U_{Y}=2$, $\mathrm{V}_{X}=18, \mathrm{~V}_{Y}=18, \mathrm{~W}_{X}=0, \mathrm{~W}_{Y}=0$, d'où $\operatorname{var}(s)=26,4$. Utilisant la valeur déjà calculée du dénominateur, Den $=14,0$, nous obtenons $\operatorname{var}\left(\tau_{\mathrm{b}}\right) \approx 26,4 / 14,0^{2} \approx 0,1347$ et $\sigma \approx 0,367$. La valeur observée du tau-b était 0,786. L'évaluation de la probabilité extrême par voie Monte Carlo (basée sur 50000 permutations aléatoires) indique une probabilité extrême $p=$ 0,022 . Pour l'approximation normale (non recommandée pour $n<30)$, nous calculons $z=(0,786-2 / 30) / 0,367 \approx 1,960$ et $p(z) \approx 0,025$.

Le traitement de l'exemple 3, sur tableau croisé de fréquences, entraîne de plus lourds calculs. Ayant déjà établi plus haut $\mathrm{U}_{X}=1650, \mathrm{U}_{Y}=2594$ et Den $\approx 3881,833$, nous totalisons $\mathrm{V}_{X}=68610, \mathrm{~V}_{Y}=161178, \mathrm{~W}_{X}=26880$ et $\mathrm{W}_{Y}=68916$, d'où $\operatorname{var}(s) \approx 100412,32$ et var $(\tau) \approx 0,006664$, d'où $\sigma \approx$ 0,0816 . L'approximation normale fournit $z=(0,2007-2 /$ 9900 ) $/ 0,0816 \approx 2,457$, d'où $p(z) \approx 0,007$; sur 50000 permutations au hasard effectuées sur la série des $Y, 350$ obtiennent une valeur $\tau \geq 0,2007$, d'où $p=0,007$ aussi.

\footnotetext{
${ }^{5}$ Des valeurs critiques exactes devraient tenir compte de tous les assortiments possibles (en termes de valeurs égales ou inégales) des $n$ valeurs observées en $X$ et en $Y$, un défi titanesque. Par exemple, pour $n=4$, on compte 4 partitions non triviales (soit $31,22,211,1111$ ), où par exemple « 31 » indique 3 valeurs égales +1 valeur autre, et 7 " compositions » (soit 31, 13, 22, 211, 121, 112, 1111), où « 31 » indique que les 3 valeurs égales sont les plus faibles, au contraire de « 13 »: d'où la nécessité de préparer $7^{2}=49$ séries critiques dans ce cas. Pour $n=5$ et 6 , nous obtenons respectivement 6 et 10 partitions, d'où 225 et 784 séries. Enfin, pour $n=10$ par exemple, il y a 41 partitions, 457 compositions et 208849 séries requises!
}

Notons ici que le khi-deux d'interaction, établi sur le tableau de l'exemple 3, vaut $\chi^{2}=22,62$, avec 15 degrés de liberté, la probabilité extrême étant $p \approx 0,093$. Nous avons donc ici le cas d'une relation monotone significative (avec $p<0,01$ ) entre $X$ et $Y$, relation que le khi-deux ne débusque pas.

\section{Discussion et conclusion}

Les variables de niveau linéaire, pour lesquelles les différences de valeurs sont mesurables (p. ex. $X_{1}-X_{2}>X_{3}-$ $X_{4}$ ), ont aussi une capacité ordinale (p. ex. $X_{1}>X_{2}$ ), de sorte que les techniques de corrélation de Pearson $(r)$, Spearman $(r s)$ et Kendall $(\tau)$ s'y appliquent toutes. Kendall et Gibbons (1990 ; voir aussi Kendall et Stuart, 1979) donnent les équivalences approximatives entre ces trois statistiques :

$$
\begin{gathered}
\tau \approx 1 / 2 \pi \sin ^{-1} r, \\
r \approx 2 \sin (\pi r s / 6),
\end{gathered}
$$

d'où d'autres équivalences peuvent être dérivées. Kendall et Gibbons présentent aussi une variante de $\tau$ dans le cas où la population $X, Y$ serait d'ordre linéaire et binormale, ce qui donne lieu à un test normal approximatif plus puissant que (6). Il reste que, pour des variables $X, Y$ proprement ordinales, par conséquent non normales, seul le tau de Kendall s'applique légitimement, et l'utilisation du $r$ ou du $r$ s ne peut y être considérée qu'abusive.

Agresti (1976) passe en revue divers indices de corrélation appliqués au traitement de données ordinales catégorielles, incluant le tau-b, le $r_{s}$ de Spearman et un indice élémentaire «gamma », $\gamma=(\mathrm{C}-\mathrm{D}) /(\mathrm{C}+\mathrm{D})$, la notation étant celle de notre formule (1). Son étude lui permet de conclure à la supériorité du tau-b, lequel serait moins affecté par des changements dans la catégorisation des variables et conserverait davantage sa convergence vers sa valeur paramétrique.

Le traitement des variables ordinales catégorielles, particulièrement pour la corrélation entre deux variables disposées en tableau, est traditionnellement problématique. Le hic tient à l'usage du khi-deux d'interaction sur ces données, tel que dénoncé par Burr (1960) et Somers (1962). Le khi-deux est un test «omnibus» applicable sur un tableau croisé de fréquences, test qui rejette l'hypothèse nulle (indépendance stochastique des variables $X$ et $Y$ ) en faveur de toutes formes de dépendance, incluant la dépendance monotone. Pour illustration, prenons le tableau simpliste suivant (chaque ligne est identifiée par une lettre) :

\begin{tabular}{ccccc}
\hline$a$ & 2 & 0 & 0 & 0 \\
\hline $\mathrm{b}$ & 0 & 2 & 0 & 0 \\
$\mathrm{c}$ & 0 & 0 & 2 & 0 \\
$\mathrm{~d}$ & 0 & 0 & 0 & 2 \\
\hline
\end{tabular}

Tel que présenté et en supposant que les rangées et colonnes sont en ordre (croissant) des variables, le tableau de fréquences $4 \times 4$ génère une valeur $\tau_{b}=1$. Les rangées dénotées «a,,$\langle\mathrm{b} »,\langle\mathrm{C} »$ et $« \mathrm{~d} »$ peuvent aussi être 
permutées, ce de $4 !=24$ façons, ce qui donne lieu aux valeurs tb suivantes, chacune associée à sa probabilité extrême :

Comme on voit, les 24 réarrangements de ce petit tableau produisent jusqu'à 7 valeurs distinctes de corrélation ordinale $^{6}$, tandis que tous aboutissent à un seul et même khi-deux d'interaction, soit $\chi^{2}=24,00$, doté de 9 degrés de liberté et de

\begin{tabular}{|c|c|c|c|c|c|c|c|}
\hline$\tau_{b}=$ & 1 & $2 / 3$ & $1 / 3$ & 0 & $-1 / 3$ & $-2 / 3$ & -1 \\
\hline \multirow{7}{*}{ 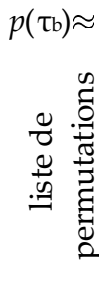 } & \multirow{7}{*}{$\begin{array}{l}0,0004 \\
\text { abcd }\end{array}$} & 0,019 & 0,161 & 0,533 & 0,161 & 0,019 & \multirow{7}{*}{$\begin{array}{l}0,0004 \\
\text { dcba }\end{array}$} \\
\hline & & abdc & $a c d b$ & adcb & bdca & cdba & \\
\hline & & acbd & $a d b c$ & bcda & cbda & dbca & \\
\hline & & bacd & badc & bdac & cdab & dcab & \\
\hline & & & bcad & $c a d b$ & dacb & & \\
\hline & & & cabd & cbad & dbac & & \\
\hline & & & & dabc & & & \\
\hline
\end{tabular}

probabilité $p \approx 0,004$. Conclure, sur la foi d'un test khi-deux, qu'il y a ou qu'il n'y a pas de corrélation dans un tableau croisé de fréquences serait donc commettre une grande imprudence.

Pour l'étude de corrélation de variables ordinales, catégorisées ou non, la technique de Kendall apparaît incontournable, et le traitement par le khi-deux ne peut être vu que comme un pis-aller dangereux.

\section{Références}

Agresti, A. (1976). The effect of category choice on some ordinal measures of association. Journal of the American Statistical Association, 71, 49-55.

Barlow, R.E., Bartholomew, D.J., Bremmer, J.M., Brunk, H.D. (1972). Statistical inference under order restrictions. New York : Wiley.

Best, D.J. (1973). Extended tables for Kendall's tau. Biometrika, 60, 429-430.
Burr, E.J. (1960). The distribution of Kendall's score $S$ for a pair of tied rankings. Biometrika, 47, 151-171.

Edgington, E.S. (1980). Randomization tests. New York: Marcel Dekker.

Jonckeere, A.R. (1954). A distribution-free k-sample test against ordered alternatives. Biometrika, 41, 133-145.

Kendall, M., et Gibbons, J.D. (1990). Rank correlation methods (5e édition). New York : Oxford University Press.

Kendall, M, Stuart, A. ( 1979). The advanced theory of statistics. Volume 2. Inference and relationship (4e édition). New York: Macmillan.

Laurencelle, L. (1993). Deux tests de variation monotone pour l'analyse de variance. Lettres Statistiques, 9, 69-78.

Laurencelle, L., Dupuis, F.A. (2000). Tables statistiques expliquées et appliquées (2e édition). Québec: Le Griffon d'argile.

Laurencelle, L. (2001). Hasard, nombres aléatoires et méthode Monte Carlo. Québec : Presses de l'Université du Québec.

Laurencelle, L. (2005). Un indice d'association entre deux variables continues. Lettres Statistiques, 12, 81-98.

Mukerjee, H. (1988). Order restricted inference in a repeated measure model. Biometrika, 75, 616-617.

Sillito, G.P. (1947). The distribution of Kendall's $\tau$ coefficient of rank correlation in rankings containing ties. Biometrika, 34, 36-40.

Siegel, S., Castellan, N.J. (1988). Nonparamtric statistics for the behavioral sciences (2e édition). New York : McGraw-Hill.

Somers, R.H. (1962). A new asymmetric measure of association for ordinal variables. American sociological review, 27, 799-811.

Valz, P.D., McLeod, A.I., Thompson, M.E. (1995). Cumulant generating function and tail probability approximations for Kendall's score with tied rankings. Annals of statistics, 23, 144-160.

Article received May 5 $5^{\text {th }}, 2009$

Article accepted September 19 , 2009

Appendices follows.

6 Par comparaison, les permutations des 8 données individuelles, au nombre de $8 ! /(2 !)^{4}=2520$, donnent lieu à 31 valeurs distinctes de tb et à 21 valeurs du $r$ s de Spearman. 
Annexe. Valeurs critiques du $\tau$ de Kendall (n valeurs distinctes en $\mathrm{X}$ et en $\mathrm{Y}$ )

\begin{tabular}{|c|c|c|c|c|c|c|c|c|c|c|c|}
\hline $\mathrm{n}$ & 0,10 & 0,05 & 0,025 & 0,01 & 0,005 & $n$ & 0,10 & 0,05 & 0,025 & 0,01 & 0,005 \\
\hline 3 & - & - & $-\quad-$ & - & & 52 & 0.124 & 0.158 & 0.189 & 0.223 & 0.246 \\
\hline 4 & 1 & 1 & $-\quad-$ & - & & 53 & 0.123 & 0.157 & 0.187 & 0.221 & 0.244 \\
\hline 5 & 0.800 & 0.800 & 11 & - & & 54 & 0.122 & 0.156 & 0.185 & 0.219 & 0.241 \\
\hline 6 & 0.600 & 0.733 & 0.867 & 0.867 & 1 & 55 & 0.121 & 0.154 & 0.182 & 0.216 & 0.239 \\
\hline 7 & 0.524 & 0.619 & 0.714 & 0.810 & 0.905 & 56 & 0.119 & 0.152 & 0.181 & 0.214 & 0.236 \\
\hline 8 & 0.429 & 0.571 & 0.643 & 0.714 & 0.786 & 57 & 0.118 & 0.152 & 0.179 & 0.212 & 0.234 \\
\hline 9 & 0.389 & 0.500 & 0.556 & 0.667 & 0.722 & 58 & 0.117 & 0.149 & 0.177 & 0.210 & 0.232 \\
\hline 10 & 0.378 & 0.467 & 0.511 & 0.600 & 0.644 & 59 & 0.116 & 0.148 & 0.176 & 0.209 & 0.230 \\
\hline 11 & 0.345 & 0.418 & 0.491 & 0.563 & 0.600 & 60 & 0.115 & 0.147 & 0.174 & 0.207 & 0.228 \\
\hline 12 & 0.303 & 0.394 & 0.455 & 0.545 & 0.576 & 61 & 0.114 & 0.145 & 0.173 & 0.204 & 0.226 \\
\hline 13 & 0.308 & 0.359 & 0.436 & 0.513 & 0.563 & 62 & 0.113 & 0.144 & 0.172 & 0.203 & 0.224 \\
\hline 14 & 0.275 & 0.363 & 0.407 & 0.473 & 0.516 & 63 & 0.112 & 0.143 & 0.171 & 0.201 & 0.223 \\
\hline 15 & 0.276 & 0.333 & 0.390 & 0.467 & 0.505 & 64 & 0.111 & 0.142 & 0.169 & 0.199 & 0.220 \\
\hline 16 & 0.250 & 0.317 & 0.383 & 0.433 & 0.483 & 65 & 0.110 & 0.140 & 0.167 & 0.198 & 0.218 \\
\hline 17 & 0.250 & 0.309 & 0.368 & 0.426 & 0.471 & 66 & 0.110 & 0.139 & 0.166 & 0.196 & 0.217 \\
\hline 18 & 0.242 & 0.294 & 0.346 & 0.412 & 0.451 & 67 & 0.108 & 0.139 & 0.164 & 0.195 & 0.215 \\
\hline 19 & 0.228 & 0.287 & 0.333 & 0.392 & 0.439 & 68 & 0.107 & 0.137 & 0.163 & 0.193 & 0.213 \\
\hline 20 & 0.221 & 0.274 & 0.326 & 0.379 & 0.421 & 69 & 0.107 & 0.136 & 0.162 & 0.192 & 0.211 \\
\hline 21 & 0.210 & 0.267 & 0.314 & 0.371 & 0.410 & 70 & 0.106 & 0.135 & 0.161 & 0.190 & 0.210 \\
\hline 22 & 0.203 & 0.264 & 0.307 & 0.359 & 0.394 & 71 & 0.105 & 0.134 & 0.160 & 0.189 & 0.209 \\
\hline 23 & 0.202 & 0.257 & 0.296 & 0.352 & 0.391 & 72 & 0.104 & 0.133 & 0.158 & 0.188 & 0.207 \\
\hline 24 & 0.196 & 0.246 & 0.290 & 0.341 & 0.377 & 73 & 0.104 & 0.132 & 0.158 & 0.186 & 0.205 \\
\hline 25 & 0.193 & 0.240 & 0.287 & 0.333 & 0.367 & 74 & 0.103 & 0.131 & 0.156 & 0.185 & 0.204 \\
\hline 26 & 0.188 & 0.237 & 0.280 & 0.329 & 0.360 & 75 & 0.102 & 0.130 & 0.155 & 0.183 & 0.203 \\
\hline 27 & 0.179 & 0.231 & 0.271 & 0.322 & 0.356 & 76 & 0.101 & 0.129 & 0.154 & 0.182 & 0.201 \\
\hline 28 & 0.180 & 0.228 & 0.265 & 0.312 & 0.344 & 77 & 0.100 & 0.129 & 0.153 & 0.181 & 0.200 \\
\hline 29 & 0.172 & 0.222 & 0.261 & 0.310 & 0.340 & 78 & 0.100 & 0.128 & 0.152 & 0.179 & 0.199 \\
\hline 30 & 0.172 & 0.218 & 0.255 & 0.301 & 0.333 & 79 & 0.099 & 0.127 & 0.151 & 0.179 & 0.197 \\
\hline 31 & 0.166 & 0.213 & 0.252 & 0.295 & 0.325 & 80 & 0.098 & 0.126 & 0.150 & 0.177 & 0.196 \\
\hline 32 & 0.165 & 0.210 & 0.246 & 0.290 & 0.323 & 81 & 0.098 & 0.125 & 0.149 & 0.176 & 0.195 \\
\hline 33 & 0.163 & 0.205 & 0.242 & 0.288 & 0.314 & 82 & 0.097 & 0.124 & 0.148 & 0.175 & 0.194 \\
\hline 34 & 0.159 & 0.201 & 0.237 & 0.280 & 0.312 & 83 & 0.097 & 0.124 & 0.147 & 0.174 & 0.192 \\
\hline 35 & 0.156 & 0.197 & 0.234 & 0.277 & 0.304 & 84 & 0.096 & 0.123 & 0.146 & 0.173 & 0.191 \\
\hline 36 & 0.152 & 0.194 & 0.232 & 0.273 & 0.302 & 85 & 0.095 & 0.122 & 0.145 & 0.172 & 0.190 \\
\hline 37 & 0.150 & 0.192 & 0.228 & 0.267 & 0.297 & 86 & 0.095 & 0.121 & 0.144 & 0.171 & 0.189 \\
\hline 38 & 0.149 & 0.189 & 0.223 & 0.263 & 0.292 & 87 & 0.094 & 0.121 & 0.144 & 0.170 & 0.187 \\
\hline 39 & 0.147 & 0.188 & 0.220 & 0.260 & 0.287 & 88 & 0.094 & 0.120 & 0.143 & 0.169 & 0.187 \\
\hline 40 & 0.144 & 0.185 & 0.218 & 0.256 & 0.285 & 89 & 0.093 & 0.119 & 0.141 & 0.168 & 0.185 \\
\hline 41 & 0.141 & 0.180 & 0.215 & 0.254 & 0.280 & 90 & 0.093 & 0.119 & 0.141 & 0.167 & 0.185 \\
\hline 42 & 0.141 & 0.178 & 0.213 & 0.250 & 0.275 & 91 & 0.092 & 0.117 & 0.140 & 0.166 & 0.183 \\
\hline 43 & 0.138 & 0.176 & 0.209 & 0.247 & 0.274 & 92 & 0.091 & 0.117 & 0.139 & 0.165 & 0.182 \\
\hline 44 & 0.137 & 0.173 & 0.207 & 0.243 & 0.268 & 93 & 0.091 & 0.116 & 0.138 & 0.164 & 0.181 \\
\hline 45 & 0.135 & 0.172 & 0.204 & 0.240 & 0.267 & 94 & 0.090 & 0.116 & 0.137 & 0.163 & 0.180 \\
\hline 46 & 0.132 & 0.169 & 0.202 & 0.239 & 0.264 & 95 & 0.090 & 0.115 & 0.137 & 0.162 & 0.179 \\
\hline 47 & 0.132 & 0.167 & 0.199 & 0.236 & 0.260 & 96 & 0.089 & 0.114 & 0.136 & 0.161 & 0.179 \\
\hline 48 & 0.129 & 0.167 & 0.197 & 0.232 & 0.257 & 97 & 0.089 & 0.114 & 0.135 & 0.160 & 0.177 \\
\hline 49 & 0.129 & 0.163 & 0.196 & 0.230 & 0.253 & 98 & 0.089 & 0.113 & 0.135 & 0.160 & 0.177 \\
\hline 50 & 0.127 & 0.162 & 0.192 & 0.228 & 0.25 & 99 & 0.088 & 0.113 & 0.134 & 0.159 & 0.175 \\
\hline 51 & 0.126 & 0.161 & 0.191 & 0.225 & 0.249 & 100 & 0.087 & 0.112 & 0.133 & 0.158 & 0.1 \\
\hline
\end{tabular}

